\title{
Factors related to the effectiveness of variable stiffness colonoscope: Results of a multivariate analysis
}

\author{
Javier Sola-Vera, Francisco Uceda, Alicia Brotons, Jesús Sáez, Eva Girona, Estefanía Pérez, \\ María Dolores Picó, Concepción Grau and Narciso Vázquez
}

\author{
Department of Digestive Medicine. Hospital General Universitario de Elche. Elche, Alicante. Spain
}

\begin{abstract}
Background: Various studies and two meta-analysis have shown that a variable stiffness colonoscope improves cecal intubation rate. However, there are few studies on how this colonoscope should be used.

Objective: The aim of this study was to identify factors related to the advancement of the colonoscope when the variable stiffness function is activated.

Methods: Prospective study enrolling consecutive patients referred for colonoscopy. The variable stiffness colonoscope (Olympus CF-H180DI/L®) was used. We performed univariate and multivariate analyses of factors associated with the success of the variable stiffness function.

Results: After the data inclusion period, 260 patients were analyzed. The variable stiffness function was used most in the proximal colon segments (ascending and transverse colon $85 \%$; descending/sigmoid colon $15.2 \%)$. The body mass index was lower in patients in whom the endoscope advanced after activating the variable stiffness than those in which it could not be advanced $\left(25.9 \pm 4.8\right.$ vs. $\left.28.3 \pm 5.4 \mathrm{~kg} / \mathrm{m}^{2}, \mathrm{p}=0.009\right)$. The endoscope advanced less frequently when the stiffness function was activated in the ascending colon versus activation in other segments of the colon (25\% vs. $64.5 \%$ ascending colon vs. other segments; $p<$ $0.05)$. In the multivariate analysis, only the colon segment in which the variable stiffness was activated was an independent predictor of advancement of the colonoscope.

Conclusions: The variable stiffness function is effective, allowing the colonoscope advancement especially when applied in the transverse colon, descending colon and sigmoid. However, when used in the ascending colon it has a lower effectiveness.
\end{abstract}

Key words: Endoscopy. Colonoscopy. Variable stiffness.

Received: 09-01-2013

Accepted: $13-12-2013$

Correspondence: Javier Sola-Vera-Sánchez. Department of Digestive Medicine. Hospital General Universitario de Elche. Camí de l'Almazara, 11. 03203 Elche. Alicante, Spain

e-mail: solavera_jav@gva.es
Sola-Vera J, Uceda F, Brotons A, Sáez J, Girona E, Pérez E, Picó MD, Grau C, Vázquez N. Factors related to the effectiveness of variable stiffness colonoscope: Results of a multivariate analysis. Rev Esp Enferm Dig 2014;106:15-21.

\section{INTRODUCTION}

During colonoscopy, the existence of marked angulations and colon adherences increase the difficulty of the procedure. In order to improve the performance of the procedure, there are several possibilities, such as the use of pediatric colonoscopes (1), the use of enteroscopes (2) and, recently, the use of variable stiffness colonoscopes (3). These endoscopes combine the flexibility of a pediatric colonoscope with the stiffness of a standard adult colonoscope through a simple device that increases or decreases the insertion tube stiffness. To date, several controlled studies (4-9) and two meta-analysis $(10,11)$ have been conducted comparing this type of colonoscope with standard colonoscopes which have shown that the variable stiffness colonoscope decreases caecal intubation time and patient discomfort. However, in a previous study in our centre (12) we found that it is not always necessary to use the variable stiffness function. In that study we observed that the variable stiffness was effective, i.e. achievement of endoscope advancement, in $66 \%$ of cases in which it was used. Knowing the situations where the activation of the variable stiffness is effective may help the endoscopist to optimize the use of this function. The main objective of the present study was to identify factors related to the advancement of the colonoscope when the variable stiffness function is activated. The secondary objective was to examine factors related to the use of the stiffness function. 


\section{MATERIALS AND METHODS}

Observational prospective (comparative) cohort study in which factors related to the advancement of the colonoscope when variable stiffness function was activated were analyzed. A study was performed in a 6-months period, which included consecutive patients over 18 years of age referred to the endoscopy unit for colonoscopy.

Patients in which a sigmoidoscopy was requested were excluded from the final analysis, as well as those with colostomy, impassable stenosis of the colon and those with poor colon cleansing which precluded colonoscopy completion. Patients with a pacemaker or implantable defibrillator were also excluded because it is a formal contraindication to the use of the ScopeGuide ${ }^{\mathrm{TM}}$, Olympus ${ }^{\mathrm{TM}}$. All patients received preparation with standard intestinal cleansing. In order to reduce result variability, all colonoscopies included in this study were performed by two doctors and two nurses, all with broad experience in digestive endoscopy (> 5,000 previous colonoscopies). A variable stiffness colonoscope was used (Olympus CF-H180AL, working length $168 \mathrm{~cm}$; outer diameter $13.2 \mathrm{~mm}$ ). This endoscope has a distinctive characteristic: A dial at the base of the handle. Rotating the dial causes increases tension on an internal cable, which leads to increased stiffness of the endoscope. The dial can be moved from position 0 (minimum stiffness) to position 3 (maximum stiffness) through intermediate positions 1 and 2 . The study was conducted in one endoscopy room. Colonoscopies were performed without sedation when there was no anesthesiologist and under deep sedation when an anesthesiologist was present.

\section{Colonoscopy technique}

The procedure was initiated with the patient in the left lateral decubitus position. The nurse introduced the endoscope following the instructions of the endoscopist handling the colonoscope. The variable stiffness function was activated as follows: The colonoscope was introduced in variable stiffness position " 0 ". The variable stiffness function was always used in the same way, that is, directly from position 0 to position 3 . The variable stiffness function was only activated when the endoscopist found it necessary, that is, when a loop appeared that prevented the slightest endoscope advance. Under these circumstances, before activating the variable stiffness, the nurse straightened the endoscope and manually compressed the abdomen, attempting to advance the colonoscope again. When no advance was possible despite the manual compression, the colonoscope was again fully straightened, after which the stiffness was changed to position " 3 ". In this position the colonoscope was reintroduced until advancement was achieved. If another loop formed at another point, the stiffness was returned to the " 0 " position, the endoscope straightened and the stiffness was again activated in the same manner. During withdrawal, the stiffness was set to "0". The active stiffness function was considered successful when it permitted the advancement of the endoscope tip, whether immediately or not. In cases where activation of the variable stiffness did not allow the advance of the endoscope we proceeded to make changes in patient position to achieve the advancement of the endoscope. To precisely locate the colon segments where the variable stiffness was activated, an electromagnetic guidance system was used (Scope Guide ${ }^{\mathrm{TM}}$, Olympus ${ }^{\mathrm{TM}}$ ). This system allows the virtual display of the form and position of the endoscope on a separate screen, without using fluoroscopy. In this way it is possible to identify the segment of the colon where a loop is formed.

For practical purposes, when stiffness was activated at the splenic flexure it was considered to be activated in the transverse colon as in most cases the tip of the endoscope was placed at the angle closest to the transverse colon. Also, when stiffness was activated at the hepatic flexure it was considered to be activated in the ascending colon as in most cases the tip of the endoscope was placed at the angle closest to the ascending colon. Demographic data, weight, height, body mass index, previous surgery, indication for colonoscopy, presence of diverticula, distance reached by colonoscopy, as well as cecal intubation time and total duration of the colonoscopy were registered. Cecal intubation time was measured with the stopwatch function of the screen, from the moment the endoscope was inserted through the anus until the tip of the colonoscope touched the cecum. The segment of the colon where variable stiffness was activated, the success of the task of advancement with the variable stiffness colonoscope, the need for manual compression, and the difficulty in performing the colonoscopy according to the endoscopist were registered. The latter parameter was measured through a visual analogue scale from 0 to 100 , where 0 was no difficulty and 100 was maximum difficulty. In patients who received no sedation, the pain perceived by the patient during the procedure was registered immediately after the colonoscopy. A visual analogue scale (VAS) from 0 to $100 \mathrm{~mm}$ was used. On that scale, the absence of pain corresponds to the point 0 and the maximum tolerable pain corresponds to point 100 . This parameter was registered by nurses, who invited the patient to place a mark on printed 100 $\mathrm{mm}$ line. The study was approved by the local IRB (study ENDORIVA01). All patients signed the informed consent form prior to participating in the study.

\section{Statistical methods}

We performed a descriptive analysis of the various parameters. We performed a univariate analysis of factors related to the use (comparing the patients where variable stiffness was activated with those where it was not acti- 
vated) and effectiveness of variable stiffness (comparing the patients in which the endoscope advanced after activating this function with those in which it did not advance). A multivariate study of factors associated with the success of the variable stiffness, i.e., the advancement of the endoscope after activating this function, was performed. Statistically significant parameters in the univariate analysis as well as clinically significant parameters were included in a multivariate binary logistic regression. Calculations were carried out with SPSS Statistical Package 17.0 (SPSS, Chicago, Ill). A $p$ value of less than 0.05 was considered significant. Sample size: The classical formula from Free$\operatorname{man}[\mathrm{n}=10 *(\mathrm{k}+1)]$ was applied for the calculation of the sample size in a logistic regression. According to this formula the sample size must be about ten times the number of independent variables plus one Freeman DH. (Applied categorical data analysis New York: Marcel Dekker Inc., 1987). In our study we assumed a maximum of 9 independent variables in the model. Thus $\mathrm{n}=10 *(9+1)=100$ cases. Assuming that variable stiffness would be used in $40 \%$ of all patients, a total of 250 patients were needed. A $10 \%$ was added in order to compensate for any losses.

\section{RESULTS}

A total of 280 patients were evaluated during the study period. Twenty patients were excluded for the following reasons: Fourteen patients in whom a rectoscopy, rectosigmoidoscopy or left colonoscopy was requested, three patients with impassable stenosis of the colon (one due to a constricting neoplasm, one after radiotherapy and one due to diverticulosis) and three patients with colostomy. Ultimately, two hundred and sixty patients were included in the study.

Table I shows the characteristics of patients included in the study. Among the parameters that indicate a difficult colonoscopy, $24 \%$ of patients were obese, $10 \%$ had a history of pelvic surgery and $10 \%$ a history of incomplete colonoscopy. History of pelvic radiation therapy were not collected in this study. Reasons for undergoing colonoscopy were: Follow-up of polyps/cancer (33.1\%), rectal bleeding $(21.2 \%)$, change in bowel movements $(9.2 \%)$, anemia $(7.7 \%)$, radiological findings $(6.9 \%)$, family history of colon cancer $(6.5 \%)$, inflammatory bowel disease (suspected or follow up) (5\%), abdominal pain (3.5\%), other $(6.2 \%)$.

Table II shows the parameters related to the colonoscopy procedure. The cecal intubation rate was somewhat low $(90 \%)$, probably because $54.6 \%$ of the procedures were performed without sedation. Thus, the percentage was $95.9 \%$ in patients sedated versus $85.8 \%$ in non-sedated patients. Cecal intubation time was $5.2 \pm 2.6$ minutes $(4$ \pm 1.8 minutes when variable stiffness was not activated and $6.8 \pm 2.7$ minutes when variable stiffness was activated). The pain perceived by the patient was recorded in
Table I. Patient characteristics. The quantitative variables are shown as mean and standard deviation

\begin{tabular}{ll}
\hline Age (years) & $58 \pm 16.6$ \\
Sex (M/F) & $145 / 115$ \\
Weight $(\mathrm{kg})$ & $78.8 \pm 16.3$ \\
Body mass index $\left(\mathrm{kg} / \mathrm{m}^{2}\right)$ & $27.1 \pm 5.3$ \\
Obesity (\%) & $63(24.2)$ \\
History of pelvic surgery (\%) & $26(10)$ \\
History of colonic surgery (\%) & $96(36.9)$ \\
Incomplete prior colonoscopy (\%) & $26(10)$ \\
Diverticulosis (\%) & $73(28.1)$ \\
\hline
\end{tabular}

109 non-sedated patients. Pain assessment by the patients was $62.2 \pm 25.3$ on a scale of 100 . Note that variable stiffness was used on 132 occasions in 117 patients, i.e. was used in $45 \%$ of patients, and was effective in achieving the advancement of the endoscope in 63 of these 132 cases, representing a $47.7 \%$ of the occasions when it was activated. In cases in which the variable stiffness was not effective other ancillary maneuvers were used, basically changes in patient position and repeated insertion/withdrawal of the endoscope, together with changes in the area where the abdominal pressure was applied.

Table III shows the factors related to the activation of variable stiffness by the endoscopist. Of all the factors involved, a previous incomplete colonoscopy, difficulty in performing the colonoscopy in the opinion of the endoscopist and pain perceived by the patient were associated with the use of variable stiffness. No relationship was found

\section{Table II. Main results related to the procedure}

\begin{tabular}{|c|c|}
\hline $\begin{array}{l}\text { Examinations under sedation/total } \\
\text { examinations }(\%)\end{array}$ & $118 / 260(45.4)$ \\
\hline Caecal intubation percentage & 90 \\
\hline Caecal intubation time (min) & $5.2 \pm 2.6$ \\
\hline Total duration of colonoscopy (min) & $14.2 \pm 7.9$ \\
\hline Change in position of patient (\%) & $73(28.1)$ \\
\hline Manual compression (\%) & $176(67.7)$ \\
\hline Difficulty of procedure (VAS 0-100 mm) & $35,1 \pm 26,3$ \\
\hline Pain (VAS 0-100 mm)* & $62,2 \pm 25,3$ \\
\hline $\begin{array}{l}\text { Number of patients where variable stiffness } \\
\text { was activated/total number of patients (\%) }\end{array}$ & $117 / 260(45)$ \\
\hline $\begin{array}{l}\text { Number of times variable stiffness was } \\
\text { activated }\end{array}$ & 132 \\
\hline Variable stiffness efficacy $(\%)^{\star *}$ & $63 / 132(47,7)$ \\
\hline
\end{tabular}


Table III. Factors related to the use of variable stiffness

\begin{tabular}{|c|c|c|c|}
\hline & $\begin{array}{l}\text { Use of variable stiffness } \\
(n=117)\end{array}$ & $\begin{array}{l}\text { Non use of variable } \\
\text { stiffness }(n=143)\end{array}$ & $p$ \\
\hline Age (yrs) & $58.6 \pm 15.9$ & $57.6 \pm 17.2$ & 0.6 \\
\hline $\begin{array}{l}\text { Sex } \\
\text { Male (\%) } \\
\text { Female (\%) }\end{array}$ & $\begin{array}{l}64(54.7) \\
53(45.3)\end{array}$ & $\begin{array}{l}81(56.6) \\
62(43.4)\end{array}$ & 0.7 \\
\hline Obesity (\%) & $32(28.3)$ & $31(22.1)$ & 0.2 \\
\hline BMI $\left(\mathrm{kg} / \mathrm{m}^{2}\right)$ & $27.1 \pm 5.2$ & $27 \pm 5.4$ & 0.9 \\
\hline Diverticulosis (\%) & $34(27.1)$ & $39(27.3)$ & 0.7 \\
\hline Previous pelvic surgery (\%) & $10(8.5)$ & $16(11.2)$ & 0.4 \\
\hline Previous colonic surgery (\%) & 49 (41.9) & $47(32.9)$ & 0.1 \\
\hline Previous incomplete colonoscopy (\%) & $28(23.9)$ & $10(10.5)$ & 0.004 \\
\hline Deep sedation (\%) & $52(44.1)$ & $66(46.2)$ & 0.8 \\
\hline Previous incomplete colonoscopy (\%) & $51.9 \pm 24.3$ & $21.3 \pm 18$ & $<0.001$ \\
\hline Pain (VAS 0-100 mm)* & $69.5 \pm 24.8$ & $56.6 \pm 24.4$ & 0.008 \\
\hline
\end{tabular}

BMI: Body mass index. VAS: Visual analog scale. *Pain was registered only in non-sedated patients.

between variable stiffness use and obesity, colon or pelvic surgery history, diverticulosis, or in relation to age, sex, or colonoscopy performed with sedation or not. The variable stiffness was used more often in the proximal segments of the colon. Thus, of the 132 times it was used, in 7 (5.3\%) cases it was used in the sigmoid colon, in $13(9.8 \%)$ cases in the descending colon, $56(42.4 \%)$ times in the transverse colon and $56(42.4 \%)$ times in the ascending colon.

Table IV lists the factors related to the success of variable stiffness, i.e., advancement of the endoscope after enabling this function. Of these, only the body mass index (BMI) and colon segment where the stiffness was activated were significantly associated with the advancement of the endoscope. Thus, BMI was lower in patients in whom the maneuver was successful compared to patients who failed $\left(25.9 \pm 4.8 v s .28 .3 \pm 5.4 \mathrm{~kg} / \mathrm{m}^{2} ; \mathrm{p}=0.009\right)$. Furthermore, we observed that the effectiveness of the variable stiffness was significantly lower when used in ascending colon when compared to other segments of the colon (Table V).

In order to identify independent factors on the effectiveness of variable stiffness, a multivariate analysis was performed which included the significant factors of the univariate analysis (BMI and colon segment) as well as other factors that may influence the progression of the endoscope after activation of the stiffness. As shown

Table IV. Parameters related to the advancement of the endoscope after activating variable stiffness

\begin{tabular}{llll}
\hline & Advancement $(n=63)^{\star}$ & Non advancement $(n=69)$ & $p$ \\
\hline Age (yrs) & $58.2 \pm 16.9$ & 60.415 .4 & 0.4 \\
Sex & & & 0.3 \\
Male (\%) & $30(47.6)$ & $39(56.5)$ & \\
Female (\%) & $33(52.4)$ & $30(43.5)$ & 0.07 \\
Obesity (\%) & $13(21)$ & $23(35.4)$ & 0.009 \\
BMl (kg/m²) & $25.9 \pm 4.8$ & $28.3 \pm 5.4$ & 0.5 \\
Diverticulosis (\%) & $17(27)$ & $22(31.9)$ & 0.3 \\
Previous pelvic surgery (\%) & $7(11.1)$ & $4(5.8)$ & 0.4 \\
Previous colonic surgery (\%) & $29(46)$ & $27(39.1)$ & 0.9 \\
Previous incomplete colonoscopy (\%) & $15(23.8)$ & $17(24.6)$ & 0.9 \\
Deep sedation (\%) & $35(55.5)$ & $38(55.1)$ & \\
\hline
\end{tabular}

BMI: Body mass index. *Variable stiffness was used on 132 occasions in 117 patients, and was effective in achieving the advancement of the endoscope in 63 of these 132 occasions. 
in table VI, only the colon segment in which stiffness was activated remained as an independent predictor of advancement of the endoscope: Sigmoid colon compared to ascending colon OR 11.1 (CI $95 \%$ 1.1-108.4), descending colon compared to ascending OR 3.9 (CI $95 \% 1.1$ 14.4) and transverse colon compared to ascending OR 5.4 (CI $95 \%$ 2.4-12.4).

\section{DISCUSSION}

Different situations may occur during colonoscopy, such as sigmoid colon adherences or serious loop formation, which hinder the procedure. Various methods have been considered in order to resolve these difficulties, such as the use of pediatric colonoscopes (1) or elements to increase the stiffness of the endoscope, such as rigid cables inserted through the biopsy channel. However, these systems are cumbersome and not widely used. Also, the pediatric colonoscope may be useful to pass through a stiff sigmoid loop, but it presents an inconvenience as its lack of stiffness leads to the formation of loops. For the last few years variable stiffness colonoscopes have been available (3) that combine the properties of the flexibility of the pediatric colonoscope with the stiffness of an adult colonoscope. This system has the advantage of being easy to activate, and allowing the stiffness to vary as needed during the colonoscopy (4).

Various studies have been completed comparing standard colonoscopes with variable stiffness colonoscopes (4-9). A meta-analysis (10) that included 7 randomized studies showed that the percentage of cecal intubation was greater with variable stiffness colonoscopes when compared to standard colonoscopes, with less abdominal pain and reduced sedation needed, without differences related to the duration of cecal intubation and use of auxiliary tasks (manual compression and posture changes). A sec-

\begin{tabular}{|c|c|c|}
\hline & $\begin{array}{l}\text { Use of variable } \\
\text { stiffness }(n=132)^{*}\end{array}$ & Advancement (\%) \\
\hline Sigmoid colon & 7 & $5(71.4)$ \\
\hline Descending colon & 13 & $7(53.8)$ \\
\hline Transverse colon & 56 & $37(66.1)$ \\
\hline Ascending colon & 56 & $14(25)^{* *}$ \\
\hline
\end{tabular}

ond recent meta-analysis (11) has also shown a greater percentage of cecal intubation with variable stiffness colonoscopes as compared to standard colonoscopes, a reduction in auxiliary maneuvers without a difference in cecal intubation time.

However, in a randomized study performed at our centre (12) we found that not all cases require the use of variable stiffness. Thus, in this study variable stiffness was used in only $32.1 \%$ of patients. Moreover, variable stiffness was effective in this study, achieving scope advancement in $66.7 \%$ of cases in which it was activated, not being effective in the other cases. The knowledge of the situations in which variable stiffness is useful may help the endoscopist to optimize the use of variable stiffness function of modern colonoscopes. Thus, it seemed interesting to design a study focused on assessing the use and factors related to the effectiveness of variable stiffness, since we have not found any study in the literature specifically designed to assess these factors.

One of the controversial issues regarding the use of variable stiffness is how it should be used, since there are few comparative studies on how best to use this function of the colonoscope. In the study by Hsieh et al. (13) com-

Table VI. Multivariate analysis of factors associated with the effectiveness of variable stiffness. The reference category for the variable colonic segment is ascending colon

\begin{tabular}{llll}
\hline & OR & IC 95\% & $p$ \\
\hline Age & 0.988 & $0.962-1.015$ & 0.3 \\
Sex (male) & 0.977 & $0.424-2.250$ & 0.7 \\
BMI & 0.954 & $0.880-1.034$ & 0.2 \\
Diverticulosis & 1.035 & $0.426-2.515$ & 0.8 \\
Previous pelvic surgery & 1.808 & $0.413-7.921$ & 0.4 \\
Deep sedation & 0.747 & $0.324-1.722$ & 0.7 \\
Colonic segment: & & & \\
$\quad$ Sigmoid & 11.143 & $1.146-108.375$ & 0.038 \\
$\quad$ Descending & 3.906 & $1.063-14.380$ & 0.040 \\
$\quad$ Transverse & 5.425 & $2.380-12.366$ & $<0.001$ \\
\hline
\end{tabular}

BMl: Body mass index. 
paring different methods of activating the variable stiffness function, there were no significant differences in the rate of cecal intubation or cecal intubation time between the routine activation of the stiffness in the descending colon with respect to its activation only when deemed necessary, although the latter form of employment was more beneficial in a subgroup of patients. In the present study the variable stiffness has been used only when deemed necessary and it has been used in a restrictive manner, i.e. not used routinely but only when no advance was possible despite manual compression. This justifies the fact that the percentage of use of variable stiffness in this study (45\%) is somewhat lower than in other published studies, which ranged between $61 \%$ and $76 \%$ (7-9). In our clinical practice we use the variable stiffness as described in this study because we believe it is the way you get maximum return from this function, as suggested by other authors (14).

In terms of factors related to the use of variable stiffness in our study, we observed that these factors do not relate to the characteristics of the patients, such as age, sex or constitutional parameters, but only relates to a prior incomplete colonoscopy, difficulty in performing the colonoscopy in the opinion of the endoscopist and pain perceived by the patient These parameter reflects in some way that colonoscopy is more difficult, a fact that was expected, since, as mentioned above, variable stiffness function is used when other measures (such as abdominal pressure) failed.

In most studies, the variable stiffness has been used more in the descending colon and sigmoid, whereas in the present study it has been used more in proximal segments of the colon. This is because a proper abdominal pressure by expert support staff suffices to pass the sigmoid and descending colon in most cases, without resorting to further ancillary maneuvers. However, in the transverse colon, hepatic flexure and ascending colon, auxiliary maneuvers are less effective, as noted in the study by Heigh et al. (15). This study assessed the efficacy of various auxiliary maneuvers during colonoscopy, including variable stiffness. Less maneuver success was noted when the colonoscope tip was in the transverse colon $(61.1 \%)$, hepatic flexure $(52 \%)$ and ascending colon $(41.7 \%)$ compared to elsewhere in the colon.

The most relevant finding in this study is that the effectiveness of variable stiffness function is related, in univariate analysis, to constitutional parameters, such as BMI, as well as the colon segment in which this function is used. However, in multivariate analysis the colon segment remains as the only independent factor associated with the success of variable stiffness. Thus, the probability of success of this maneuver is 11 times higher when activated in the sigmoid colon, 4 times higher when activated in the descending colon and 5 times higher when activated in the transverse colon, when compared to ascending colon. In other words, the maneuver is only effective in $25 \%$ of cases when activated in ascending colon compared with $70 \%, 66 \%$ and $54 \%$ when activated in sigma, transverse and descending colon, respectively.

The limitations of this study are that it was conducted in a tertiary hospital by experienced endoscopists and nurses so that the results cannot be generalized to other types of facilities and personnel. Also, the study was conducted with electromagnetic guidance system, which helps to know at all times where the tip of the endoscope is. This system is not available in most centers.

In conclusion, the variable stiffness function can improve the advance of the colonoscope in about half the cases when used after failed manual compression. One must keep in mind that its effectiveness depends largely on the colon segment in which this function is used. As the activation is performed very easily, we believe it is worth to activate this feature when the colonoscope does not progress in the distal colon before taking further auxiliary maneuvers. However, when the colonoscope does not progress in the ascending colon we should be prepared to take other ancillary measures, since in these circumstances the variable stiffness function probably will not improve advancement.

\section{REFERENCES}

1. Bat L, Williams CB. Usefulness of pediatric colonoscopes in adult colonoscopy. Gastrointest Endosc 1989;35:329-32.

2. Kaltenbach T, Soetikno R, Friedland S. Use of a double balloon enteroscope facilitates caecal intubation after incomplete colonoscopy with a standard colonoscope. Dig Liver Dis 2006;38:921-5.

3. Brooker JC, Saunders BP, Shah SG, Williams CB. A new variable stiffness colonoscope makes colonoscopy easier: A randomised controlled trial. Gut 2000;46:801-5

4. Shumaker DA, Zaman A, Katon RM. A randomized controlled trial in a training institution comparing a pediatric variable stiffness colonoscope, a pediatric colonoscope, and an adult colonoscope. Gastrointest Endosc 2002;55:172-9.

5. Kaffes AJ, Mishra A, Ding SL, Hope R, Williams SJ, Gillespie PE, et al. A prospective trial of variable stiffness pediatric vs. standard instrument colonoscopy. Gastrointest Endosc 2003;58:685-9.

6. Lee DW, Li AC, Ko CW, Chu DW, Chan KC, Poon CM, et al. Use of a variable-stiffness colonoscope decreases the dose of patient-controlled sedation during colonoscopy: A randomized comparison of 3 colonoscopes. Gastrointest Endosc 2007;65:424-9.

7. Rex DK. Effect of variable stiffness colonoscopes on caecal intubation times for routine colonoscopy by an experienced examiner in sedated patients. Endoscopy 2001;33:60-4.

8. Yoshikawa I, Honda H, Nagata K, Kanda K, Yamasaki T, Kume K, et al. Variable stiffness colonoscopes are associated with less pain during colonoscopy in non-sedated patients. Am J Gastroenterol 2002;97:3052-5.

9. Al-Shurieki SH, Marshall JB. Is the variable-stiffness paediatric colonoscope more effective than a standard adult colonoscope for outpatient adult colonoscopy? A randomised controlled trial. Dig Liver Dis 2005;37:698-704.

10. Othman MO, Bradley AG, Choudhary A, Hoffman RM, Roy PK. Variable stiffness colonoscope versus regular adult colonoscope: Metaanalysis of randomized controlled trials. Endoscopy 2009;41:17-24.

11. Xie Q, Chen B, Liu L, Gan H. Does the variable-stiffness colonoscope makes colonoscopy easier? A meta-analysis of the efficacy of the variable stiffness colonoscope compared with the standard adult colonoscope BMC Gastroenterology 2012;12:151. 
12. Sola-Vera J, Uceda F, Brotons A, Sáez J, Girona E, Pérez E, et al. Does the use of a variable stiffness colonoscope offer advantages during colonoscopy under deep sedation? Results of a randomized trial. Eur J Gastroenterol Hepatol 2011;23:593-7.

13. Hsieh YH, Zhou AL, Lin HJ. Comparing different methods of activating the variable stiffness function of a pediatric variable stiffness colonoscope. J Chin Med Assoc 2008;71:23-9.
14. Ginsberg GG. Colonoscopy with the variable stiffness colonoscope. Gastrointest Endosc 2003;58:579-84.

15. Heigh RI, DiBaise JK, Prechel JA, Horn BJ, San Miguel S, Heigh EG, et al. Use of an electromagnetic colonoscope to assess maneuvers associated with cecal intubation. BMC Gastroenterol 2009;9:24. 